MAGNETIC source imaging revealed that the topographic representation in the somatosensory cortex of the face area in upper extremity amputees was shifted an average of $1.5 \mathrm{~cm}$ toward the area that would normally receive input from the now absent nerves supplying the hand and fingers. Observed alterations provide evidence for extensive plastic reorganization in the adult human cortex following nervous system injury, but they are not a sufficient cause of the phantom phenomenon termed 'facial remapping'.

Key words: Plasticity; Cortex; Phantom; Somatotopy; Magnetoencephalography (MEG); Magnetic source imaging

\section{Extensive reorganization of the somatosensory cortex in adult humans after nervous system injury}

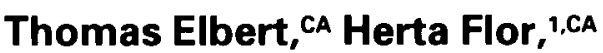 \\ Niels Birbaumer, ${ }^{2}$ Stefan Knecht, \\ Scott Hampson, Wolfgang Larbig ${ }^{2}$ \\ and Edward Taub ${ }^{3}$
}

\author{
Institute of Experimental Audiology and \\ Department of Neurology, University of \\ Münster, Kardinal-von-Galen-Ring 10, D-48129 \\ Münster; 'Department of Psychology, \\ Humboldt-University Berlin, Oranienburger \\ Straße 18, D-10178 Berlin; 'Institute of Medical \\ Psychology and Behavioural Neurobiology, \\ University of Tübingen, Gartenstraße 29, \\ D-72074 Tübingen, Germany; ${ }^{3}$ Department of \\ Psychology, University of Alabama at \\ Birmingham, AL 35294, USA
}

a Corresponding Authors

\section{Introduction}

Merzenich and his colleagues ${ }^{1}$ reported plastic reorganization of the primary somatosensory cortex subsequent to the amputation of individual digits in adult owl monkeys. The representation of neighbouring digits 'invaded' the cortical space that was formerly occupied by the representation of the amputated portions of the hand. In a recent study in adult macaque monkeys, Pons and coworkers ${ }^{2}$ reported massive reorganization of primary somatosensory cortex subsequent to sensory deafferentation of an entire arm 12 years earlier. The portion of the primary somatosensory cortex that had been deprived of its normal inputs from the arm anomalously responded to tactile stimulation of the face. Whereas the reorganization reported by Merzenich et al' spanned a distance of 1-2 mm, Pons et al ${ }^{2}$ observed plastic changes in the cortical map that were an order of magnitude greater $(1-2 \mathrm{~cm})$. There was a topographic isomorphism between specific facial locations of tactile stimulation and the location of evoked responses in the (deafferented) cortical area normally representing the arm and hand. In human upper extremity amputees, it has been similarly reported ${ }^{3-6}$ that touching specific localized portions of the face or the amputation stump can evoke sensations that are referred to the amputated phantom limbs and bear a constant topographic relationship to the sites of stimulation. Ramachandran et al ${ }^{+}$referred to this phenomenon as 'remapping' of the amputated limb. It has been suggested that remapping might be the perceptual correlate of a reorganization of the primary somatosensory cortex in human amputees similar to that observed in animal studies. ${ }^{5-8}$ This conceptually attractive proposal involves two linked hypotheses that require empirical evaluation: (1) cortical reorganization of the somatosensory map takes place in humans following upper extremity amputation, and, if so (2) this cortical reorganization (i.e. a central neuronal change) is responsible for facial remapping (a perceptual change). ${ }^{9}$ Based on magnetoencephalographic (MEG) and electroencephalographic (EEG) recordings, we provide evidence here that supports the first hypothesis, but not the second. The evidence for the first hypothesis can be considered an independent replication and extension of a recent study by Yang $e t$ al. ${ }^{10}$

The synchronous measurement of electric potentials and magnetic fields spanning a minimum of $1 \mathrm{~mm}^{2}$ of neuronal mass activity permits the exact localization of the cortical somatosensory map. ${ }^{11,12}$ It also permits the assessment of changes due to altered inputs, such as that produced by surgical separation of 'webbed' hands in humans ${ }^{13}$ and, by extension, that produced by the elimination of inputs resulting from limb amputa- 
tion. If the first hypothesis is correct, then the distance between the face and hand areas in the somatosensory cortex contralateral to (and representing) the intact arm should be larger than the distance between the corresponding areas in the somatosensory cortex contralateral to the amputated arm. The distances of these representations are equivalent (mean difference $<3.2$ $\mathrm{mm}$ ) for the two hemispheres in humans with two intact arms, as shown by Gallen et al ${ }^{1+}$ using the same experimental setup as the present one.

\section{Materials and Methods}

Five male subjects who reported phantom limb sensations subsequent to unilateral hand or arm amputations were included in the study (Table 1). After giving informed consent the subjects participated in a structured comprehensive interview about their amputation and the location, quality and extent of phantom pains and phantom sensations and stump-related complications. The neurological assessment included determination of sensory or motor disturbances. The presence of remapping was explored by a standardized procedure similar to that employed by Ramachandran et al. ${ }^{5}$ Beginning at the unaffected foot, all body regions were touched with a Q-tip while the patient kept his eyes closed. The patient had to report the location, quality and intensity of the perceived sensation. The instruction emphasized that sensations at all (even distant) body sites should be reported. All remapped sites and the sites of the referred phantom sensations were coded on templates; the topography of the remapped site was marked on the skin and photographed. Somatosensory detection and pain thresholds were determined at each remapped site and at the corresponding contralateral site by administering two ascending and descending series of electric and thermal stimuli. No significant side differences were observed.

Stimulation consisted of light superficial pressure applied via a pneumatic stimulator using a standard non-painful stimulation intensity as described in Ref. 11. In all subjects the following sites were stimulated: (1) the first and fifth digits of the intact hand, (2) two

Table 1. Subject characteristics

\begin{tabular}{|c|c|c|c|c|c|c|}
\hline Subject & $\begin{array}{c}\text { Site of } \\
\text { amputation }\end{array}$ & $\begin{array}{c}\text { Age } \\
\text { (years) }\end{array}$ & $\begin{array}{c}\text { Age at } \\
\text { amputation }\end{array}$ & Cause & $\begin{array}{l}\text { Invasion" } \\
(\mathrm{cm})\end{array}$ & $\begin{array}{c}\text { Phantom } \\
\text { pain }{ }^{\circ}\end{array}$ \\
\hline T37 & $\begin{array}{l}20 \mathrm{~cm} \text { below } \\
\text { right } \\
\text { shoulder }\end{array}$ & 70 & 50 & Accident & 1.0 & 0 \\
\hline T38 & $\begin{array}{l}21 \mathrm{~cm} \text { below } \\
\text { right } \\
\text { shoulder }\end{array}$ & 31 & 15 & Osteosarcoma & 1.8 & 1.7 \\
\hline T39 & $\begin{array}{l}\text { right hand } \\
\text { below wrist }\end{array}$ & 47 & 10 & Accident & 3.3 & 9.6 \\
\hline T40 & $\begin{array}{l}10 \mathrm{~cm} \text { above } \\
\text { left elbow }\end{array}$ & 34 & 23 & Accident & 1.3 & 5.2 \\
\hline T41 & $\begin{array}{l}18 \mathrm{~cm} \text { below } \\
\text { left shoulder }\end{array}$ & 49 & 42 & Osteosarcoma & 0.5 & 2.6 \\
\hline
\end{tabular}

sites in a remapped arca of the distal stump, (3) the corresponding sites on the intact arm, (4) two sites on the side of the face ipsilateral to the amputation (chin just below the corner of the mouth and $2 \mathrm{~cm}$ below the ear), (5) the same two sites on the side of the face contralateral to the amputation. At each site 240 stimuli were delivered at an average stimulation rate of $1 \mathrm{~Hz}$; site sequence was varied according to a fixed irregular order across subjects. After each train of 240 stimuli, the subjects indicated the primary and secondary location of the perceived stimulation as well as its quality and intensity.

Using a BTi Neuromagnetometer (intrinsic noise 5-7 fT/sqrt $\mathrm{Hz}$ ), magnetic fields were recorded from 37 locations over a circular concave area $(14.4 \mathrm{~cm}$ diameter) above the parietotemporal cortex contralateral to the site of the stimulation. The MEG was sampled at a rate of $297.5 \mathrm{~Hz}$ (bandpass $0.01-100 \mathrm{~Hz}$ ). Artifacts were removed by excluding a response from the average if its range exceeded $3 \mathrm{pT}$ in any of the MEG channels or $200 \mu \mathrm{V}$ in the EEG. The rejection rate varied between 3 and $15 \%$ of responses per stimulation site.

For each evoked magnetic field a single equivalent current dipole (ECD) model (best fitting local sphere) was fitted for each sampling point. For the analysis, a contiguous sequence of five sampling points spanning a time segment within the range of $50-120 \mathrm{~ms}$ was selected per stimulation site. The selection of this focal time segment was based on whether it contained: (1) the maximat RMS (root mean square across channels) with a signal to noise ratio $>2$, (2) the maximal goodness of fit $(>0.95)$ of the ECD-model to the measured field and (3) the minimal confidence volume $(<300$ $\mathrm{mm}^{3}$ ) of the ECD location. The goodness of fit and confidence volume criteria were well fulfilled for the chin and digit dipoles. However, stimulation of the upper arm and the area below the ear failed to meet the specified criteria in about half the cases and these were consequently not included in the statistical analysis.

In addition, the EEG was recorded from the vertex and four lateral sites $(\mathrm{C} 3, \mathrm{C} 4, \mathrm{~A} 1, \mathrm{~A} 2)$ referred to $\mathrm{Fz}$. In order to establish the plausibility of the sources calculated from the MEG data, the estimated ECD locations that were obtained were overlaid on the individual magnetic resonance images (MRI).

\section{Results}

Facial remapping occurred in subject T38, but not in any of the other subjects. The frequency of this phenomenon in our sample is consistent with Ramachandran's observations.

In all five subjects a reorganization of the map of the somatosensory cortex was observed. In order to quantitatively define the extent of the reorganization, the cortical receptive fields of stimulation sites on the amputation side of the body were projected on to the somatosensory map representing the intact side of the 


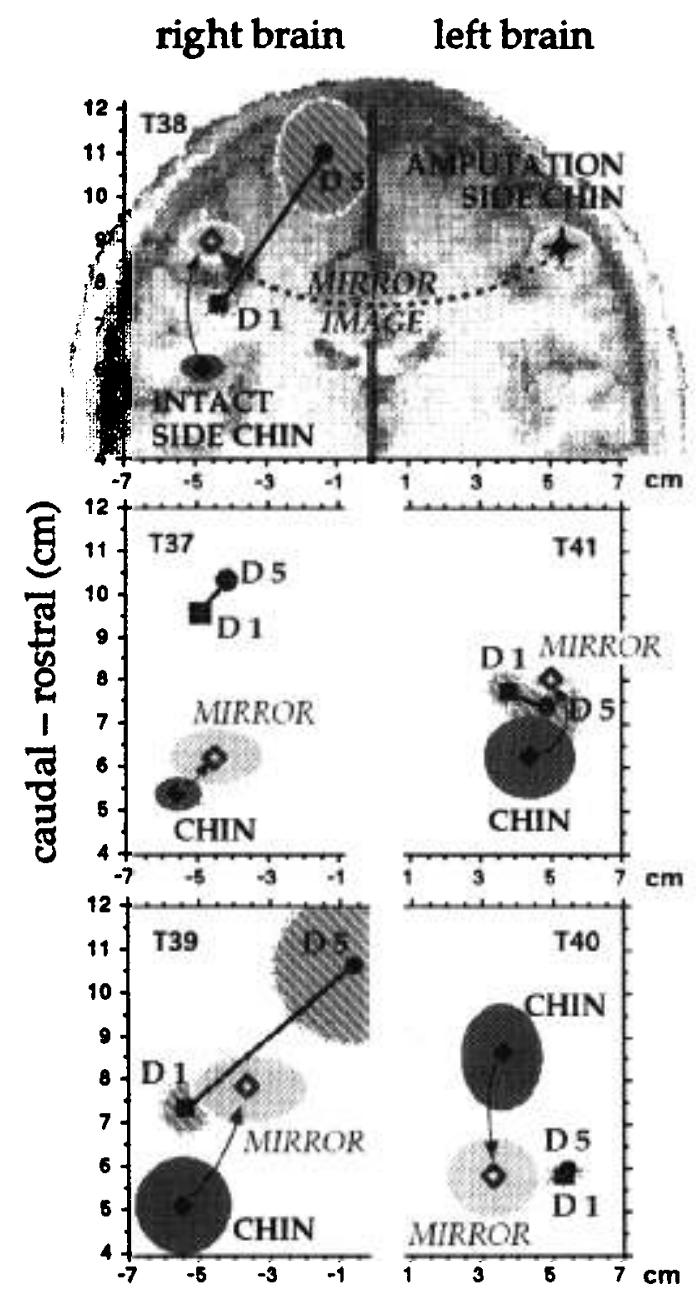

FIG. 1. The upper panel shows a coronal MRI section through the somatosensory cortices on to which are projected the dipole locations of digit one (D1 ) digit five (D5 ) and chin ( ) of the intact side of the body. The dipole location of the chin of the amputated side $(t)$ of the body was mirrored by projecting it (dashed arrow) to a corresponding location on the opposite hemisphere $(\bullet$ denotes the mirror location). The solid arrow shows the invasion of the chin area of the amputation side into the region normally representing the digits. The shaded areas represent two-dimensional projections of the confidence volumes of the dipole locations. Because the anterior-posterior axes are not represented in this two-dimensional section, there is an appearance of overlap in confidence volumes which did not actually exist. For the remaining four cases (lower panels) only the dipole locations in the hemisphere representing the intact side of the body are shown.

body (i.e. as a 'mirror-image'). The Euclidean distances were then measured between the cortical representations of the fifth and the first digits, respectively, and the chin of the intact side, and between the cortical representations of the fifth and the first digits of the intact side and the 'mirror image' of the chin on the amputation side. These distances were then compared to determine the extent to which the face area on the amputation side had invaded the cortical amputation zone (formerly the hand area). An analysis of variance revealed that the mirror image of the chin of the amputation side was significantly closer to the representation of the fingers than the receptive field of the chin of the intact side $(\mathrm{F}(1,4)=15.82, p<0.01$; one-tailed $)$. Whereas the average distances of the representations of the first and fifth digits to the chin representation of the intact side were $3.46 \mathrm{~cm}$ (s.d. $=1.69$ ) and $5.09 \mathrm{~cm}$ (s.d. = 2.30), respectively, the distances of the two digits from the mirror image of the chin of the amputation side were $2.38 \mathrm{~cm}$ (s.d. = 1.01) and $3.27 \mathrm{~cm}(\mathrm{~s} . d .=1.59)$. This 'invasion' of the amputation zone by the chin representation was present clearly in four of the five subjects (Fig. 1), and in the fifth subject (T37) a substantial shift toward the amputation zone occurred. In another of the subjects (T40, Fig. 1), the MRI indicated a deformity in the configuration of the central sulcus which led to a localization of the digits inferior to the chin location. In this patient the ECD locations of the amputation stump and upper arm 'moved' not to a rostral but to a caudal location (i.e. also approached the finger location).

The central portion of Figure 2 shows the MRI and MEG dipole overlays for the upper arm and the digits of patient T37. Pre-recording examination had indicated that remapping was present in the proximal stump of this subject with a specific correspondence of stump stimulation sites and referred sensations in the different phantom fingers. The distance between the representations of the fingers and upper arm in the somatosensory cortex representing the intact side was $3.7 \mathrm{~cm}$ (digit 5) and $2.6 \mathrm{~cm}$ (digit 1) compared with 1.2 $\mathrm{cm}$ from the dipole locations of either finger to the mirror image of the amputation-side chin, a mean decrease of $2.0 \mathrm{~cm}$. The upper part of Figure 2 indicates that the magnetic responses resulting from stimulation of the amputation stump and upper arm have no resemblance to responses resulting from stimulation of the intact upper arm but mimic the responses resulting from stimulation of the intact digits.

In non-amputated controls, evoked potentials recorded from the two hemispheres show almost identical shapes, magnitudes, and latencies for corresponding locations. ${ }^{15}$ In patient T37, evoked potentials recorded after stimulation of the remapped amputation stump are in every respect comparable to those recorded after stimulation of an intact digit, but are substantially different from those recorded subsequent to stimulation of the corresponding area of the intact upper arm (lower part of Figure 2). Similarly, the peak amplitudes of the EEG responses to stimulation of the amputation-side chin was nearly twice the response to stimulation of the intact-side chin and was similar to the peak amplitude of the response to stimulation of the intact digits.

\section{Conclusion}

This study provides further evidence that extensive cortical reorganization similar to that described by Pons et al' following somatosensory deafferentation in monkeys also occurs and persists in humans following 


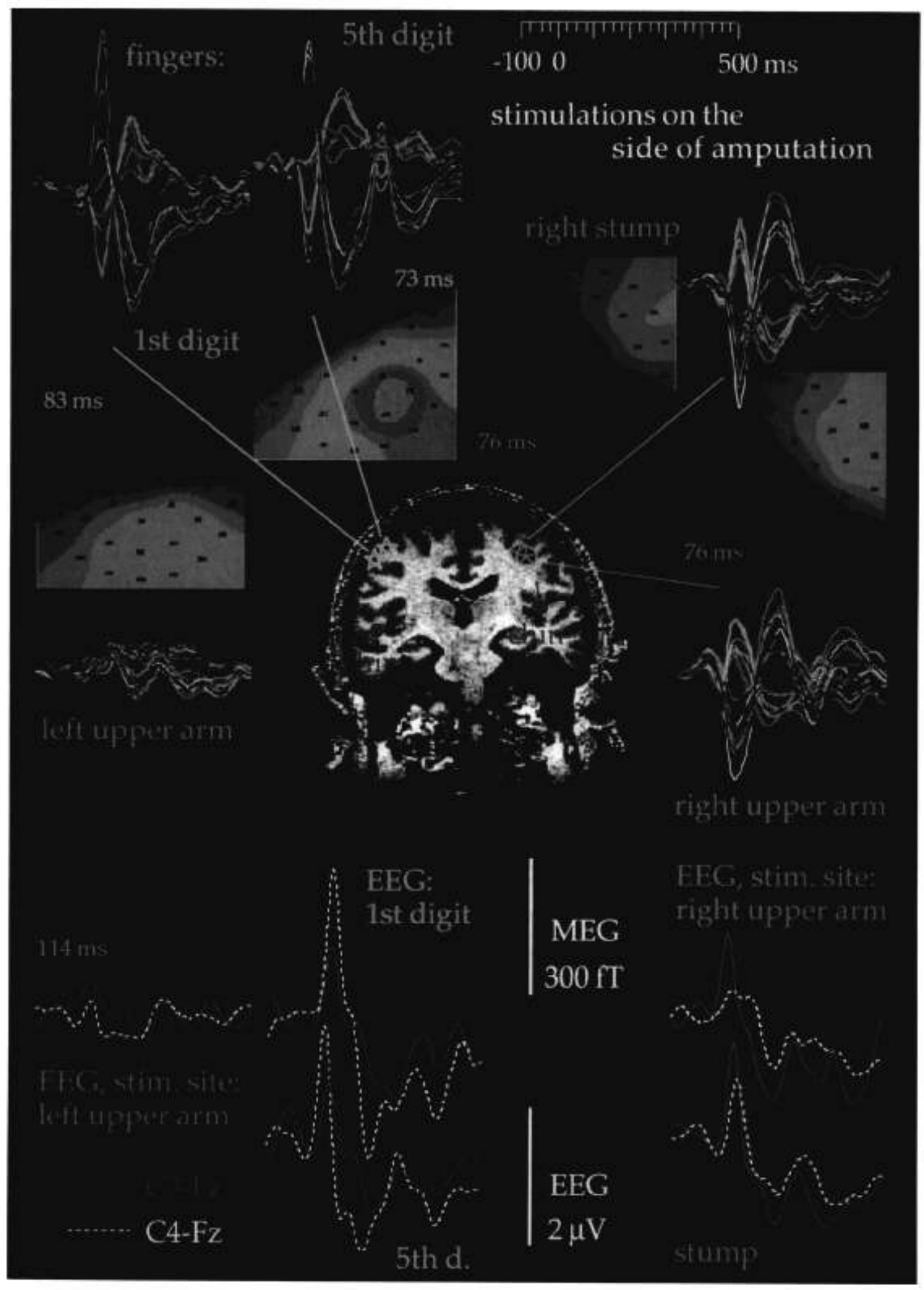

FIG. 2. The central part of the figure shows the overlay of a coronal MR image on to which are projected the equivalent current dipoles for patient T37 produced by stimulation of the stump and upper arm (right arm) and first digit, fifth digit and upper arm of the intact upper extremity (left arm). Surrounding the MR image in the upper part of the figure are inserts showing for each site the averaged magnetic fields from $37 \mathrm{channels} \mathrm{(different}$ colours for each channel overlaid on one another); beneath each magnetic field representation is shown a contour plot for the peak in the $50-120$ ms range. In the lower part of the figure the evoked EEG responses are presented for the same stimulation sites (recorded from C3, left hemisphere and $\mathrm{C4}$, right hemisphere); the sampling and processing of the EEG signals was identical to the procedure for the MEG data. 
upper extremity amputation. In all five subjects tested in this study, the cortical chin representation of the amputated side of the body invaded (four subjects) or substantially shifted toward (one subject) the area normally representing the digits. The absolute magnitude of the shift of the cortical face representation into the former cortical hand area is approximately the same in this study after upper extremity amputation in humans $(1.1-1.8 \mathrm{~cm})$ as after upper extremity somatosensory deafferentation in macaque monkeys (1.2-1.8 $\mathrm{cm})^{2}$

In this investigation, change in cortical reorganization was not correlated with reports of facial remapping. Thus, while it remains possible that cortical reorganization is necessary for the remapping of a phantom hand on the surface of the face after upper extremity amputation, it is clearly not sufficient to explain this perceptual phenomenon. Further work is needed to determine the role of cortical reorganization in the recovery from the shock that follows central nervous system damage and the rehabilitation of function. ${ }^{1.16}$

\section{References}

1. Merzenich MM, Nelson RJ, Stryker MP et al. J Comp Neurol 224, 591-605 (1984).

2. Pons TP, Garraghty AK, Ommaya AK et al. Science 252, 1857-1860 (1991).

3. Cronholm B. Acta Psychiat Neurol Scand 72, 1-310 (1951).

4. Ramachandran VS, Rogers-Ramachandran D and Stewart M. Science 258 1159-1160 (1992).

5. Ramachandran VS, Stewart $M$ and Rogers-Ramachandran D. NeuroReport 3 583-586 (1992).

6. Halligan PW, Marshall DTW, Davey J et al. NeuroReport 4, 233-236 (1993). 7. Pons TP. Science 258, 1160 (1992).

8. Katz J. Can J Psychiatry 37, 282-298 (1992).

9. Taub E. In Julesz B, Cowan G, eds. Maturational Windows and Cortical Plasticity in Human Development: is There Reason for an Optimistic View? Santa Barbara: Addison-Weslay. In press.

10. Yang TT, Gallen CC, Ramachandran VS et al. NeuroReport 5, 701-704 (1994).

11. Yang TT, Gallen CC. Schwartz BJ et al. Proc Natl ACad Sci USA 90, 3089-3102 (1993).

12. Baumgartner C, Doppelbauer A, Deecke $L$ et al. Exp Brain Res 87, 641-648 (1991).

3. Mogilner A, Grossmann JA, Ribary U et al. Proc Natl Acad Sci USA $90,3593-$ 3597 (1993).

14. Gallen CC, Sobel DF, Lewine JD et al. Radiology 187, 863-867 (1993).

15. Kaufman L, Okada Y, Brenner D et al. Int J Neurosci 15, 223-239 (1981).

16. Taub E, Miller NE, Novack TA et al. Arch Phys Med Rehab 74, 347-354 (1993).

ACKNOWLEDGEMENTS: This work was supported by grants from the Deutsche Forschungsgemeinschaft. 